\title{
AN APPLICATION OF ARTIFICIAL NEURAL NETWORK TO COMPUTE THE RESONANT FREQUENCY OF E-SHAPED COMPACT MICROSTRIP ANTENNAS
}

\author{
Ali Akdagli* — Abdurrahim Toktas \\ Ahmet Kayabasi $^{* * *}$ _ Ibrahim Develi ${ }^{* * * *}$
}

\begin{abstract}
An application of artificial neural network (ANN) based on multilayer perceptrons (MLP) to compute the resonant frequency of E-shaped compact microstrip antennas (ECMAs) is presented in this paper. The resonant frequencies of 144 ECMAs with different dimensions and electrical parameters were firstly determined by using IE3D ${ }^{(\mathrm{tm})}$ software based on the method of moments (MoM), then the ANN model for computing the resonant frequency was built by considering the simulation data. The parameters and respective resonant frequency values of 130 simulated ECMAs were employed for training and the remaining 14 ECMAs were used for testing the model. The computed resonant frequencies for training and testing by ANN were obtained with the average percentage errors (APE) of $0.257 \%$ and $0.523 \%$, respectively. The validity and accuracy of the present approach was verified on the measurement results of an ECMA fabricated in this study. Furthermore, the effects of the slots loading method over the resonant frequency were investigated to explain the relationship between the slots and resonant frequency.
\end{abstract}

K e y w or ds: compact microstrip antenna, E-shaped antenna, resonant frequency, artificial neural network (ANN)

\section{INTRODUCTION}

Because of their attractive features such as low profile, light weight, easy fabrication, low cost and conformability for mounting hosts, microstrip antennas have become popular in mobile communications. However, microstrip antennas inherently have a narrow bandwidth and low gain $[16,21,25]$. Conventional microstrip antennas with basic shapes such as rectangular, triangular and circular are relatively large to operate at the frequency band required for practical mobile applications. Present-day mobile communication systems usually require smaller antennas in order to meet the miniaturization requirements of mobile units. Therefore, size reduction or compactness has become the key consideration in antenna design for miniature wireless communication terminals. Many techniques have been proposed to effectively reduce the size of microstrip antennas [7, 18,27]. The most well-known methods, which depend upon modifying the patch geometry to reduce the resonant frequency, are slotting on the patch and ground [7], loading a shorting-pin between the patch and the ground plane [18] and using an inverted patch [27]. In fact, by using these techniques, the linear dimensions of such compact microstrip antennas (CMA) with the aforementioned basic shapes can be as small as one-third of that of corresponding conventional microstrip antennas at fixed operating frequency. The E-shaped compact microstrip antenna (ECMA) is a miniaturized antenna constructed with two identical parallel slots on the patch of rectangular microstrip antenna (RMA) by applying the slot loading method. By adjusting the slot length and slot width of the ECMA, one can obtain satisfactory performances [26].

It is well known that analytical techniques such as the cavity model [19] and the transmission line model (TLM) [2] have been successfully utilized in the analysis of conventional microstrip antennas. These methods, based on some fundamental simplifying physical assumptions regarding the radiation mechanism of antennas, are the most useful for practical design as well as providing a good intuitive explanation of microstrip antennas. However, these methods are more suitable for conventional microstrip antennas because of their regular shapes. On the other hand, powerful simulation tools involving rigorous mathematical formulation and extensive numerical procedures, such as the finite difference time domain (FDTD) method [22] and the method of moments (MoM) [10], have been successfully used to model such devices; however, the design procedure may be highly time consuming using these tools. Alternative simple ways should therefore be investigated by taking into consideration that the analysis of the microstrip patch is a complex problem because of the fringing fields at the edges. There exist several approaches which vary in accuracy and computational efforts have been proposed to analyze and design microstrip antennas. The most widely used can be

\footnotetext{
* Department of Electrical \& Electronics Engineering, Faculty of Engineering, Mersin University, 33343, Ciftlikkoy, Mersin, Turkey

** Department of Information Technologies, Mersin University, 33343, Ciftlikkoy, Mersin, Turkey

*** Department of Electronic \& Automation, Silifke-Tasucu Vocational School, Selcuk University, 33900, Silifke, Mersin, Turkey

**** Department of Electrical \& Electronics Engineering, Faculty of Engineering, Erciyes University, 38039, Kayseri, Turkey develi@erciyes.edu.tr
} 


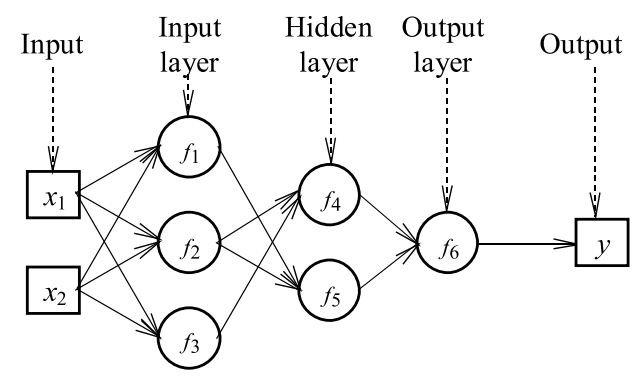

Fig. 1. ANN architecture based on the MLPs
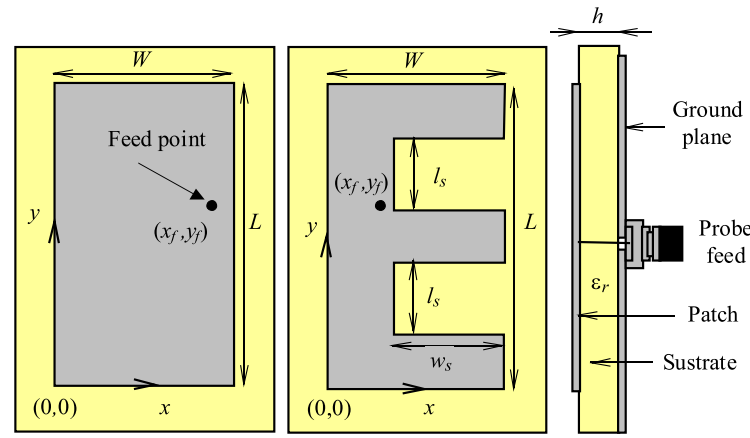

Fig. 2. Geometry of $a-R M A$ and $b-$ ECMA

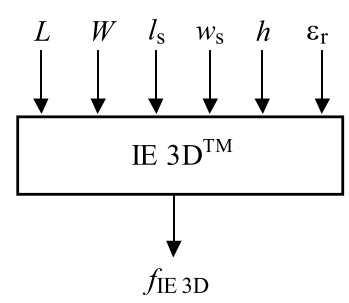

Fig. 3. Simulation process

listed as formulation methods $[1,5,24]$ and artificial intelligent systems $[4,8,15,17,20,23]$. Formulation methods are commonly derived with the aid of the optimization algorithm such as genetic, particle swarm, differential evolution etc. The most well-known artificial intelligent systems are the artificial neural network (ANN) $[4,8,11,13,15,17,20,23]$ and the adaptive neuro-fuzzy interference system (ANFIS) [13]. ANN attempts to model nonlinear problems by employing a mathematical model of the structure of the brain. The idea behind ANN models is that imitating the brain's structure of many connected processing elements enables computers to tackle tasks. During the last decade, ANN models have been increasingly used in the design of antennas, microwave devices, and circuits due to their ability and adaptability to learn, generalization, smaller information requirement, fast real-time operation, and ease of implementation features.

This work attempts to build an ANN model based on multilayer perceptrons (MLP) to compute the first resonant frequency of ECMAs. The resonant frequency values of 144 ECMAs were determined by the commercial electromagnetic simulator IE3D ${ }^{\mathrm{TM}}$ (IE3D ${ }^{\mathrm{TM}}$, version 14$)$ running on MoM. MLP was used to model the relationship between the simulated antenna parameters, which are patch dimensions and dielectric constants, and corresponding resonant frequency values. The constructed MLP model was achieved in two processes: training and testing. In order to provide the robustness of the networks, the parameters of 130 random simulated ECMAs were used to train the model and the remaining 14 were employed to test the accuracy of the model. The validity of the MLP model was verified on an ECMA fabricated in this study. Moreover, it was demonstrated that the slots into the ECMA patch lead to a reduction in resonant frequency of $36 \%$ compared to the RMA. Thus the ECMA is $61 \%$ smaller in size than the RMA. Meanwhile, the interrelation between the slots' size and the resonant frequencies and bandwidth are investigated to effectively reduce the size of the antennas.

\section{ARCHITECTURE OF ARTIFICIAL NEURAL NETWORK}

ANN is a computational model that is inspired by the function of biological neural networks. ANN consists of a group of artificial neurons which processes information over interconnection. There are many different ANN structures. Multilayer perceptrons (MLPs) $[6,11,13]$ which are successfully and commonly employed in engineering problems are preferred in this study. The MLP can be trained by using many different algorithms such as Levenberg-Marquardt (LM), backpropagation, delta-bar-delta etc. In this work, MLPs are trained with the Levenberg-Marquardt algorithm [3,9] which has the abilities of fast learning and good convergence. The MLP consists of three layers: input layer, output layer and a hidden layer, as shown in Fig. 1. Neurons in the input layer only act as buffers for distributing the input signals $x_{i}$ to neurons in the hidden layer. Each neuron $j$ in the hidden layer sums up its input signals $x_{i}$ after weighting them with the strengths of the respective connections $w_{j i}$ from the input layer and computes its output $y_{j}$ as a function $f$ of the sum, namely

$$
y_{j}=f\left(\sum w_{j i} x_{i}\right)
$$

where $f(\cdot)$ can be a simple threshold function, a sigmoid, hyperbolic tangent, a radial basis function, a purelin function etc $[11,13]$. The output of neurons in the output layer is computed similarly.

Training a network consists of adjusting the weights of the network by using one of the available learning algorithms. The learning algorithm gives the change $\Delta w_{j i}(t)$ in the weight of a connection between neurons $i$ and $j$ at time $t$. For the LM learning algorithm, the weights are updated according to the following formula

$$
w_{j i}(t+1)=w_{j i}(t)-\Delta w_{j i}(t)
$$


Table 1. Physical and electrical parameters of simulated ECMAs

\begin{tabular}{ccccccc}
\hline Number of & \multicolumn{5}{c}{ Patch Dimensions $(\mathrm{mm})$} \\
\cline { 2 - 6 } simulations & $L$ & $W$ & $l_{s}$ & $w_{s}$ & $h$ & $\varepsilon_{r}$ \\
\hline \multirow{3}{*}{$3 \times 48$} & 25.0 & 20 & $2,4,6,8$ & $4,8,12,16$ & 1.57 & $2.33,4.5,6.15$ \\
& 32.5 & 25 & $2.5,5,7.5,10$ & $5,10,15,20$ & 2.50 & $2.33,4.5,6.15$ \\
& 40.0 & 30 & $3,6,9,12$ & $6,12,20,26$ & 3.17 & $2.33,4.5,6.15$ \\
\hline
\end{tabular}

Table 2. Resonant frequencies determined by ANN for testing process

\begin{tabular}{|c|c|c|c|c|c|c|c|c|}
\hline \multicolumn{5}{|c|}{ Patch Dimensions (mm) } & \multirow[b]{2}{*}{$\varepsilon_{r}$} & \multicolumn{2}{|c|}{$\begin{array}{l}\text { Resonant } \\
\text { frequencies }(\mathrm{GHz})\end{array}$} & \multirow{2}{*}{$\begin{array}{l}\text { Percentage } \\
\text { Errors (\%) }\end{array}$} \\
\hline$L$ & $W$ & $l_{s}$ & $w_{s}$ & $h$ & & Simulated & ANN & \\
\hline 25 & 20 & 6 & 4 & 1.57 & 2.33 & 3.490 & 3.492 & 0.066 \\
\hline 25 & 20 & 4 & 8 & 1.57 & 2.33 & 2.970 & 2.972 & 0.071 \\
\hline 25 & 20 & 8 & 4 & 1.57 & 2.33 & 3.524 & 3.542 & 0.516 \\
\hline 25 & 20 & 6 & 4 & 1.57 & 4.50 & 2.587 & 2.578 & 0.332 \\
\hline 25 & 20 & 2 & 12 & 1.57 & 6.15 & 1.597 & 1.603 & 0.382 \\
\hline 32.5 & 25 & 7.5 & 5 & 2.50 & 2.33 & 2.660 & 2.675 & 0.560 \\
\hline 32.5 & 25 & 2.5 & 10 & 2.50 & 4.50 & 1.739 & 1.728 & 0.615 \\
\hline 32.5 & 25 & 7.5 & 20 & 2.50 & 4.50 & 1.170 & 1.157 & 1.103 \\
\hline 32.5 & 25 & 7.5 & 20 & 2.50 & 6.15 & 1.009 & 1.003 & 0.644 \\
\hline 40 & 30 & 3 & 20 & 3.17 & 2.33 & 1.500 & 1.492 & 0.533 \\
\hline 40 & 30 & 3 & 26 & 3.17 & 4.50 & 0.899 & 0.886 & 1.446 \\
\hline 25 & 20 & 8 & 8 & 1.57 & 4.50 & 2.310 & 2.301 & 0.403 \\
\hline 40 & 30 & 3 & 6 & 3.17 & 6.15 & 1.400 & 1.405 & 0.371 \\
\hline 40 & 30 & 9 & 12 & 3.17 & 6.15 & 1.220 & 1.223 & 0.279 \\
\hline \multicolumn{8}{|c|}{ APE } & 0.523 \\
\hline
\end{tabular}

Table 3. Results of simulation, measurement, ANN for fabricated RMA

\begin{tabular}{ccccccccccc}
\hline & \multicolumn{4}{c}{ Patch Dimensions $(\mathrm{mm})$} & \multicolumn{3}{c}{ Feed Point } & \multicolumn{3}{c}{ Resonant frequencies } \\
\cline { 1 - 8 } Antenna & $L$ & $W$ & $l_{s}$ & $w_{s}$ & $x_{f}$ & $y_{f}$ & Sim. & Mea. & ANN \\
\hline ECMA $^{*}$ & 25.00 & 20.00 & 7.47 & 13.03 & 6.45 & 14.23 & 2.400 & 2.407 & 2.396 \\
RMA & 39.85 & 32.35 & - & - & 31.07 & 26.3 & 2.400 & - & - \\
RMA & 25.00 & 20.00 & - & - & 17.64 & 16.63 & 3.759 & - & - \\
\hline
\end{tabular}

$h=1.57 \mathrm{~mm}, \varepsilon_{r}=2.33, \tan \delta=0.0012,{ }^{*}$ Rogers $^{\mathrm{TM}} \mathrm{RT} /$ duroid 5870

with

$$
\Delta w_{j i}=\left[J^{\top}(w) J(w)+\mu I\right]^{-1} J^{\top}(w) E(w)
$$

where $J, \mu, I$ and $E(w)$ are the Jacobian matrix, a constant, identity matrix, and error function, respectively. The Jacobian matrix contains the first derivatives of the errors with respect to the weights and biases. The value of $\mu$ is decreased after each successful step and is increased only when a step would increase the sum of squares of errors.

In this study, an ANN model with three layers, which are the input layer, hidden layer with three nodes and the output layer, was used. In the training process, the number of epochs, minimum gradient, momentum parameter $(\mu), \mu$ increment, $\mu$ decrement, maximum $\mu$ and seed value were selected as $250,10^{-10}, 0.0001,4,0.1,10^{10}$, 7559532 , respectively. Furthermore, tangent sigmoid, tangent sigmoid and purelin function were used in the input layer, hidden layer and output layer, respectively.

\section{DESIGN AND SIMULATION OF ECMAs}

The geometry of the RMA and ECMA is given in Fig. 2a and b. The ECMA consists of an $L \times W$ rectangular patch given in Fig. 2a with two symmetric identical slots $\left(I_{s} \times w_{s}\right.$ on a dielectric substrate with $h$ thickness on a metallic ground plane. The slots on the patch lead to an increase in the length of the patch according to TLM. Thus, the resonant frequency of the antenna can be effectively reduced.

The topology of the simulation process is illustrated in Fig. 3. It is seen that the parameters groups given in Table 1, which include the various dimensions and the electrical parameters of the ECMAs, are used to generate resonant frequency values with the aid of $\mathrm{IE}_{3} \mathrm{D}^{(\mathrm{tm})}$ software. In the simulations, the antennas were fed with a source having a Gaussian wave through a $50 \mathrm{ohm}$ probe in the vicinity of the point $x_{f}=2\left(W-w_{s}\right) / 3$ and $y_{f}=$ 


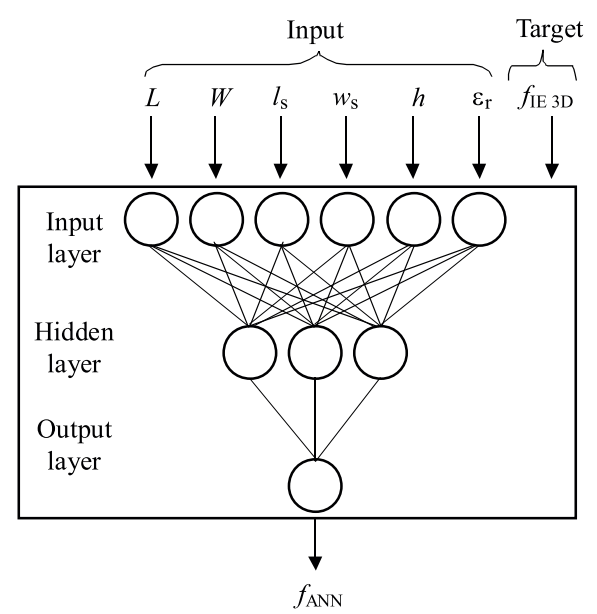

Fig. 4. Training process of ANN model

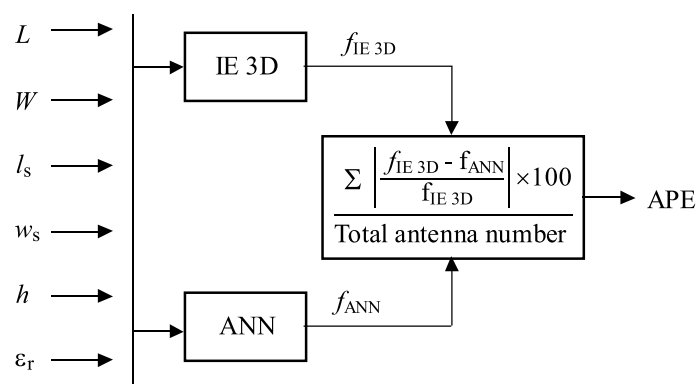

Fig. 5. The topology of calculating APE

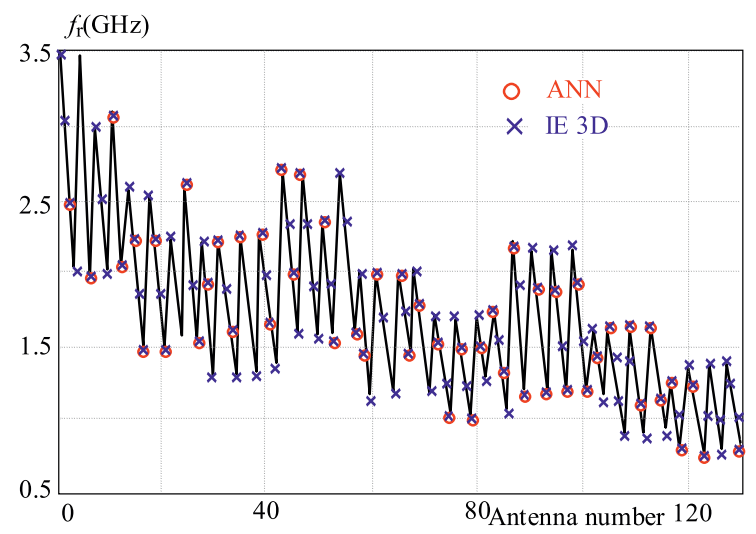

Fig. 6. Comparative results of simulation and ANN

$\left(2 L-l_{s}\right) / 3$. The maximum frequency and cell/wavelength rate were selected as $4 \mathrm{GHz}$ and $40 \mathrm{GHz}$, respectively.

The effects of the slots, dimensions on the resonant frequencies were investigated during the simulations. It was observed that slot loading on RMA to build the geometry of the ECMA results in an extension of the resonant length, and therefore the resonant frequency reduces even though the bandwidth is decreased. Furthermore, the impacts of the slot length $l_{s}$ and slot width $w_{s}$ over resonant frequency and bandwidth were sought separately, and it was inferred that slot width is more effective than the slot length in the design of an ECMA. Therefore, variation in slot length slightly changes the resonant frequency and bandwidth; otherwise an increase in the slot width leads to a decrease in resonant frequency and bandwidth.

\section{ANN MODELING}

\subsection{Training Process of ANN}

In the training process of the ANN model, the parameter groups of the ECMAs $\left(L, W, l_{s}, w_{s}, h\right.$ and $\left.\varepsilon_{r}\right)$ were introduced as input and respective simulated resonant frequency values $\left(f_{I E 3 D}\right)$ were given as a target to the ANN, as shown in Fig. 4. According to the relationship between the input and the target, the ANN model with three layers was trained to produce the resonant frequency $\left(f_{A N N}\right)$ for each parameter set of an antenna given in Table 1.

As much as 144 ECMAs were employed for training while 14 ECMAs were used for testing the ANN model. According to Fig. 5, the value of the average percentage errors (APE) for the resonant frequencies computed by the ANN model was obtained as $0.257 \%$ for the 130 ECMAs' training data. The training results of the 130 ECMAs are comparatively shown in Fig. 6, and it is seen that the simulation and training results are in very good agreement.

\subsection{Testing Process of ANN}

To verify the ANN model, 14 simulated ECMAs, which were randomly selected from a total of 144 antennas before the training process so as to represent the solution space, were used in the testing process. The computed resonant frequencies and corresponding percentage errors are tabulated in Table 2. As is seen, the resonant frequency results computed by ANN are much closer to the simulated ones. The APE was determined as $0.523 \%$ for 14 ECMAs' test data.

\section{FABRICATION OF ECMA}

In order to further investigate the validity of the present approach, an ECMA, the parameters of which were not used in the training process, operating at $2.4 \mathrm{GHz}$ was designed via IE3D ${ }^{\mathrm{TM}}$ and then fabricated on the Rogers RT/duroid 5870 substrate given in Fig. 7.
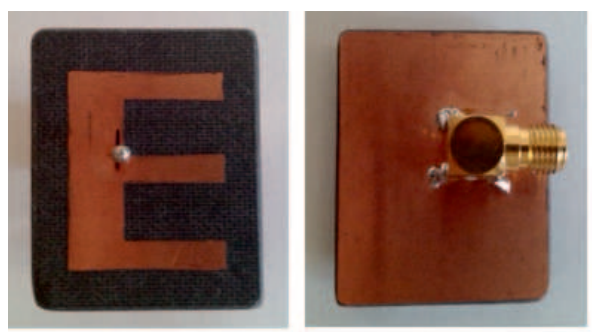

Fig. 7. Photographs of fabricated ECMA 


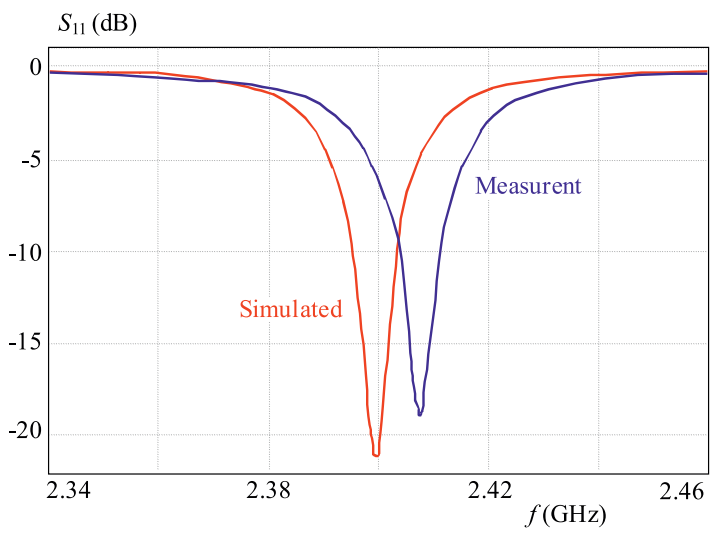

Fig. 8. Return loss $\left(s_{11}\right)$ graph of fabricated ECMA

Also two RMAs, one of them operating at the same frequency and the other with the same dimensions as the fabricated ECMA, were simulated through IE3D ${ }^{\text {TM }}$ to demonstrate that the slot loading method can be used for frequency reduction to achieve miniaturization. The

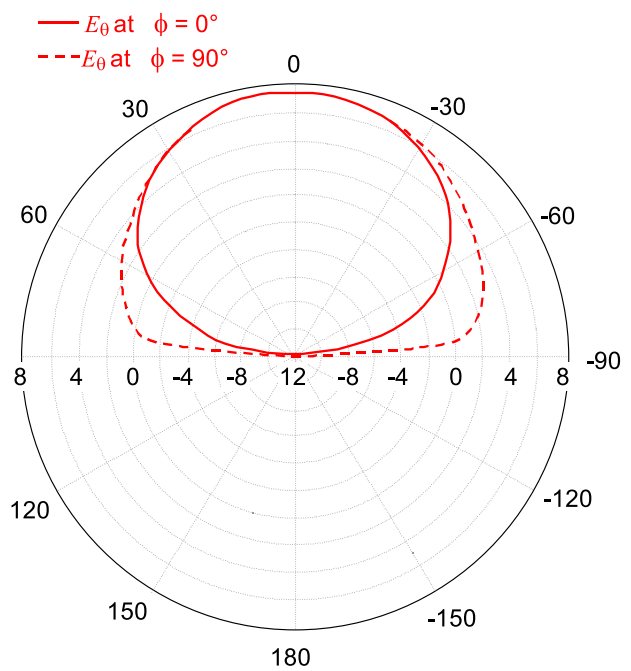

(a) $3.759 \mathrm{GHz}$ for RMA

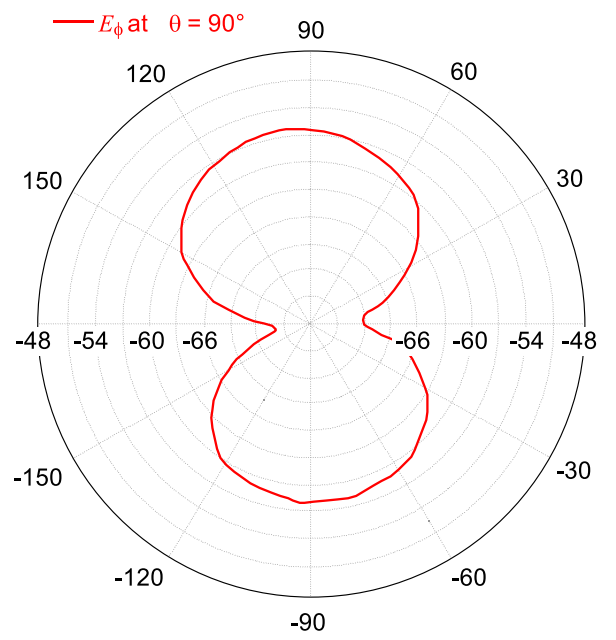

(c) $3.759 \mathrm{GHz}$ for RMA return loss of the antenna was measured with an Agilent E5071B ENA Series RF network analyzer. Fig. 8 shows a good agreement between the measured and simulated return loss curves of the ECMA, in spite of some discrepancies due mainly to substrate variations, fabrication tolerances, and feed connector misalignment. The explicit results of measurement, simulation and ANN for the fabricated ECMA and simulated RMA are comparatively given in Table 3. Moreover, it can be clearly seen that the slots in the patch of the ECMA lead to a reduction in resonant frequency of $36 \%$ compared to an RMA of the same size, so the patch size of the ECMA is $61 \%$ smaller than that of the RMA at fixed $2.4 \mathrm{GHz}$.

The simulated radiation patterns of the ECMA and RMA of the same size are given in Fig. 9. As can be seen from the figures the ECMA has a broader beamwidth than the RMA. In simulation, the maximum gain at $\phi=$ $90^{\circ}$ for the ECMA is obtained as $6.88 \mathrm{dBi}$ while it is $7.33 \mathrm{dBi}$ for the RMA. On the other hand, the half power

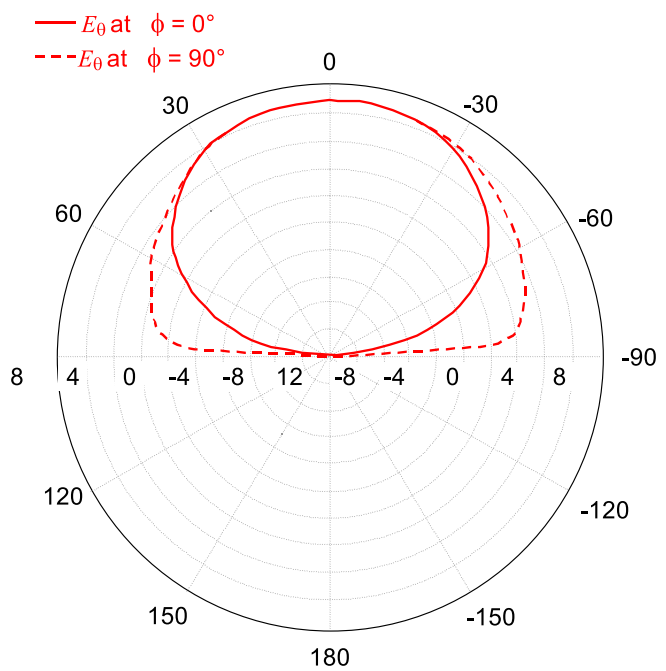

(b) $2.4 \mathrm{GHz}$ for ECMA

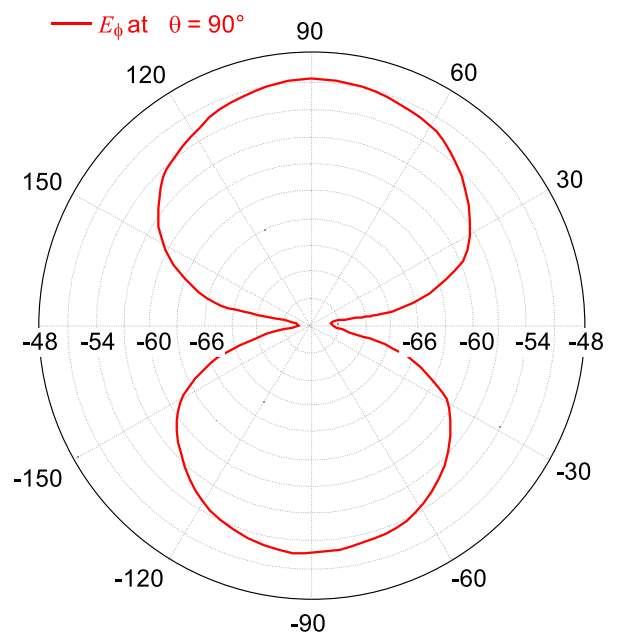

(d) $2.4 \mathrm{GHz}$ for ECMA

Fig. 9. Radiation patterns of $E_{\theta}$ at $\phi=0^{\circ}$ and $\phi=90^{\circ}$ for a - RMA and b-ECMA; Radiation patterns of $E_{\phi}$ at $\theta=0^{\circ}$ for c - RMA and $\mathrm{d}-\mathrm{ECMA}$ 
beam width is determined for the ECMA as $118.72^{\circ}$ while it is $109.36^{\circ}$ for the RMA.

\section{CONCLUSION}

In this paper, an application of ANN based on MLP for accurately calculating the resonant frequencies of ECMAs is proposed. To train and test the ANN model, 144 ECMAs with various dimensions and dielectric constants were designed and simulated by using IE3D ${ }^{\text {тм }}$ which is a commercial electromagnetic simulation tool. The parameter sets of 130 ECMAs were employed to train the proposed model and the remaining 14 ECMAs were used for testing the accuracy of the models. It was shown that the training and testing results of the ANN model were well-matched to the simulated resonant frequency results. Furthermore, an ECMA was fabricated to prove the proposed approach. In conclusion, these results substantiate that the proposed ANN model can be successfully used to determine the resonant frequencies of ECMAs without the need for any other calculations. Meanwhile, the effect of the slot sizes on the resonant frequency and bandwidth were investigated in the course of simulations. It was observed that slot width is more effective than slot length in setting the resonant frequency, and an ECMA is $61 \%$ smaller than an RMA with the same outer sizes. This clearly shows that the slot loading method can be employed for miniaturization.

\section{REFERENCES}

[1] AKDAGLi, A.-TOKTAS, A.: A Novel Expression in Calculating Resonant Frequency of H-Shaped Compact Microstrip Antennas Obtained by using Artificial Bee Colony Algorithm, Journal of Electromagnetic Waves and Applications 24 (2010), 2049-2061.

[2] Bhattacharyya, A. K.,-Garg, R.: Generalized Transmission-Line Model for Microstrip Patches, IEE Proceedings-H Microwaves Antennas and Propagation 132 (1985), 93-98.

[3] BISHOP, C. M.: Neural Networks for Pattern Recognition, Oxford University Press, London, 1995.

[4] DADGARniA, A.-HEIDARI, A. A. : A Fast Systematic Approach for Microstrip Antenna Design and Optimization using ANFIS and GA jour Journal of Electromagnetic Waves and Applications.

[5] DEShmuKH, A. A.-KUMAR, G.: Formulation of Resonant Frequency for Compact Rectangular Microstrip Antennas, Microwave and Optical Technology Letters 49 (2007), 498-501.

[6] DEVELI, I.: Application of Multilayer Perceptron Networks to Laser Diode Nonlinearity Determination for Radio-Overfibre Mobile Communications, Microwave and Optical Technology Letters 42 (2004), 425-427.

[7] DUBOst, G.-RABBAA, A.: Analysis of a Slot Microstrip Antenna, IEEE Transactions on Antennas and Propagation 34 (1986), 155-163.

[8] GUNEY, K.-SARIKAYA, N.: Input Resistance Calculation for Circular Microstrip Antennas using Adaptive Neuro-Fuzzy Inference System, International Journal of Infrared and Millimeter Waves 25 (2004), 703-716.
[9] HAGAN, M. T.-MEnHAJ, M. B.: Training Feedforward Networks with the Marquardt Algorithm, IEEE Transactions on Neural Networks 5, (1994), 989-993.

[10] HARRIngton, R. F.: Field Computation by Moment Methods, IEEE Press Series on Electromagnetic Wave Theory, Piscataway, 1993.

[11] HAYKIN, S.: Neural Networks: a Comprehensive Foundation, Macmillan College Publishing Company, New York, 1994.

12] IE3DTm, version 14, Menthor Graphics Corporation, Boeckman Road Wilsonville, OR 97070.

13] JANG, J. S. R. : Self-Learning Fuzzy Controllers based on Temporal Back Propagation, IEEE Transactions on Neural Networks 3 (1992), 714-723.

14] JANG, J. S. R.: ANFIS: Adaptive-Network-Based Fuzzy Inference System, IEEE Transactions on Systems Man and Cybernetics 23 (1993), 665-685.

15] KAYABASI, A.-BICER, M. B.-AKDAGLI, A.-TOKTAS, A. : Computing Resonant Frequency of H-Shaped Compact Microstrip Antennas Operating at UHF Band by using Artificial Neural Networks, Journal of the Faculty of Engineering and Architecture of Gazi University 26 (2011), 833-840.

16] KUMAR, G.-RAY, K. P.: Broadband Microstrip Antennas, Artech House, Boston, 2003.

17] MALATHI, P.-KUMAR, R.: On the Design of Multilayer Circular Microstrip Antenna using Artificial Neural Network, International Journal of Recent Trends in Engineering 2 (2009), $70-74$.

[18] PAN, S. C.-WONG, K. L.: Dual-Frequency Triangular Microstrip Antenna with a Shorting Pin, IEEE Transactions on Antennas and Propagation 45 (1997), 1889-1891.

19] RICHARDS, W. F.-LO, Y. T.-HARRISSON, D. D.: An Improved Theory for Microstrip Antennas and Applications, IEEE Transactions on Antennas and Propagation 29 (1981), $38-46$.

[20] SAGIROGLU, S.-GUNEY, K.: Calculation of Resonant Frequency for an Equilateral Triangular Microstrip Antenna with the use of Artificial Neural Networks, Microwave and Optical Technology Letters 14 (1997), 89-93.

[21] SAINATI, R. A.: CAD of Microstrip Antennas for Wireless Applications, Artech House, Norwood, 1996.

[22] TAFLOVE, A.: Computational Electrodynamics: The Finite-Difference Time Domain Methodpubladdr Bostonpubl Artech House, 1995.

[23] THAKARE, V. V.-SINGHAL, P.: Microstrip Antenna Design using Artificial Neural Networks, International Journal of RF and Microwave Computer-Aided Engineering 20 (2010), 76-86.

[24] TOKTAS, A.-BICER, M. B.-AKDAGLI, A.-KAYABASI, A.: Simple Formulas for Calculating Resonant Frequencies of $\mathrm{C}$ and $\mathrm{H}$ Shaped Compact Microstrip Antennas Obtained by using Artificial Bee Colony Algorithm, Journal of Electromagnetic Waves and Applications 25 (2011), 1718-1729.

[25] WONG, K. L.: Compact and Broadband Microstrip Antennas, John Wiley \& Sons, New York, 2002.

[26] YANG, F.-ZHANG, X. X.-YE, X. N.-RAHMAT-SAMII, Y.: Wide-Band E-Shaped Patch Antennas for Wireless Communications, IEEE Transactions on Antennas and Propagation 49 (2001), 1094-1100.

[27] ZHANG, Q.-FUKUOKA, Y.--ITOH, T.: Analysis of a Suspended Patch Antenna Excited by an Electromagnetically Coupled Inverted Microstrip Feed, IEEE Transactions on Antennas and Propagation 33 (1985), 895-899.

Received 6 January 2013

Ali Akdagli,Abdurrahim Toktas, Ahmet Kayabasi, biographies not supplied. Ibrahim Develi, for biography see page 43 of this volume. 